\title{
Valor Diagnóstico da Ressonância Magnética na Avaliação da Dor Lombar
}

\section{Diagnostic significance of magnetic resonance in low back pain}

\author{
Marcos Almeida-Matos ${ }^{1}$ y Maurício Santos-Gusmão²
}

1 Escola Bahiana de Medicina e Saúde Pública, Bahía, Brasil. malmeidamatos@ig.com.br

2. Santa Casa de Misericórdia da Bahia, Brasil

Recebido 9 Abril 2007/Enviado para Modificação 8 Dezembro 2007/Aprovado em 15 Janeiro 2008

\section{RESUMO}

Objetivo O objetivo deste estudo foi avaliar o valor diagnóstico da ressonância magnética-RM nas doenças da coluna lombar entre observadores com variados níveis de experiência.

Metodos Foram selecionados 20 exames de RM que foram classificados como normais [10] ou alterados [10]. Esta classificação foi chamada de "padrão" e comparada com uma segunda realizada por 15 observadores de variados níveis de experiência, denominada "teste".

Resultados Para identificação de alterações o índice Kappa foi 0,56 com sensibilidade de $96,7 \%$ e especificidade de 59,3\%. Quando consideramos o acerto diagnóstico, obteve-se índice kappa de 0,19 para hérnia discal; 0,15 para doença degenerativa discal; 0,03 para discite infecciosa; e 0,24 para espondilartrose.

Conclusões Os autores concluíram que a RM é exame de imagem com alta sensibilidade e moderada especificidade para identificação de alterações anatômicas da coluna lombar, contudo não permite que avaliadores com pouca experiência esclareçam satisfatoriamente o diagnóstico etiológico. Por este motivo, acreditamos que a solicitada rotineiramente deste exame por não-especialistas ocasiona aumento desnecessário de custos e erros diagnósticos e terapêuticos freqüentes.

Palavras Chaves: Dor lombar, diagnóstico, ressonância magnética (fonte: DeCS, BIREME).

\section{ABSTRACT}

Objective This study was aimed at assessing the diagnostic value of magnetic resonance (MR) in spinal diseases amongst observers having several levels of experience.

Methods A selection was made of $20 \mathrm{MR}$ exams which were classified as being normal [10] or altered [10]; such classification was called "standard" and then compared to a second classification made by 15 observers having varying levels of experience, called "test".

Results The Kappa index was 0,56 (96,7\% sensitivity and 59,3\% specificity) for identifying alterations. A 0,19 Kappa index was obtained for disk hernia when diagnostic 
correctness was considered, 0,15 for degenerative disk disease, 0,03 for infectious diskitis and 0,24 for spondylarthrosis.

Conclusions The authors concluded that MR is an image exam having high sensitivity and moderate specificity for identifying anatomical alterations of the spinal column; however, it does not allow assessors having little experience to satisfactorily explain an aetiological diagnosis. It is thus believed that routine requests for this exam by non-specialists leads to an unnecessary increase in costs and frequent diagnostic and therapeutic errors.

Key Words: diagnosis, magnetic resonance (source: MeSH, NLM).

\section{RESUMEN}

Valor diagnóstico de la resonancia magnética en la evaluación del dolor lumbar

Objetivo El estudio se propuso evaluar el valor diagnóstico de la resonancia magnética-RM, en enfermedades de la columna lumbar, entre observadores con varios niveles de experiencia.

Métodos Se seleccionaron 20 exámenes de RM de los cuales 10 fueron clasificados como normales y 10 como alterados. Esta clasificación fue tomada como patrón y fue comparada con una segunda clasificación o "prueba" realizada por 15 observadores con varios niveles de experiencia.

Resultados En la identificación de alteraciones el índice Kappa fue de 0,56 con 96,7 $\%$ de sensibilidad y 59,3\% de especificidad. Con diagnóstico correcto el índice de Kappa fue de 0,19 en hernia discal; 0,15 en enfermedad discal degenerativa; 0,03 en disquitis infecciosa; y 0,24 en espondiloartrosis.

Conclusiones La RM es un examen de imagen con alta sensibilidad y moderada especificidad para identificar alteraciones anatómicas de la columna lumbar; sinb embargo, no permite que evaluadores con escasa experiencia esclarezcan satisfactoriamente el diagnóstico etiológico. Por esta razón, el uso cotidiano de este examen por no especialistas incrementa innecesariamente los costos y los errores diagnósticos y terapéuticos.

Palabras Clave: diagnóstico, resonancia magnética (fuente: DeCS, BIREME).

A ressonância magnética (RM), na última década, se tornou exame complementar muito solicitado para diagnóstico etiológico das doenças da coluna lombar substituindo tomografia computadorizada, discografia e mielografia (1). Isto pode ser comprovado pelo aumento no percentual de solicitações deste exame, que passou de 22 \% em 1987 para 75 \% dos casos em 1990; com isto, os custos aumentaram de 70 para 176 milhões de dólares neste mesmo período (2).

Como vantagem a RM é exame não invasivo e demonstrou excelente sensibilidade no diagnóstico das hérnias discais, se tornando exame de escolha 
para compressão radicular (3); entretanto, sua confiabilidade continua sendo assunto controverso na literatura, especialmente por ser exame interpretadordependente. O objetivo deste estudo é avaliar o valor diagnóstico da RM em doenças da coluna lombar entre observadores com variados níveis de experiência.

\section{MÉTODOS}

O trabalho foi aprovado pela comissão de ética em pesquisa da Santa Casa de Misericórdia da Bahia-Escola Bahiana de Medicina e Saúde Pública e foi realizado entre os meses de abril e maio de 2004. Todos os exames de RM foram obtidos de pacientes matriculados no ambulatório de coluna do Hospital Santa IzabelEscola Bahiana de Medicina e Saúde Pública, sendo que a identidade dos indivíduos foi omitida nestes exames.

Os autores selecionaram 20 exames que foram avaliados previamente por três consultores: dois ortopedistas (um especialista em coluna vertebral) e um radiologista especializado em tecido músculo-esquelético. Os consultores avaliaram a qualidade das imagens e a presença ou ausência de alterações sugestivas de doença. Todos os exames foram considerados de boa qualidade. Dez entre os 20 exames foram considerados suspeitos para alterações patológicas na coluna lombar e 10 foram considerados não-suspeitos (sem alterações). Esta classificação foi chamada de "padrão" e foi utilizada para comparação com uma segunda classificação realizada por observadores selecionados, denominados "teste".

As alterações encontradas nos exames suspeitos foram: doença degenerativa discal, hérnia discal, espondilartrose e discite infecciosa, conforme Tabela 1. Os exames foram fotografados em alta resolução com máquina digital de 4,5 megapixels e, a seguir, diagramados em programa específico de imagem digital.

Tabela 1. Alterações encontradas nos exames classificados como "padrão"

\begin{tabular}{lc}
\hline \multicolumn{1}{c}{ Diagnóstico } & Número de exames \\
\hline Năo-suspeitos & 10 \\
Suspeitos para doença degenerativa & 3 \\
Suspeitos para hérnia discal & 4 \\
Suspeitos para espondilartrose & 1 \\
Suspeitos para discite infecciosa & 2 \\
\hline Total & 20
\end{tabular}

As imagens obtidas foram ordenadas aleatoriamente e submetidas à avaliação de 15 observadores (testes) sob a forma de projeção em multimídia. Os observadores foram 11 residentes de ortopedia, sendo seis do segundo ano e 
cinco do terceiro ano; e quatro preceptores da residência de ortopedia e traumatologia com título de especialista. Todos receberam folha de resposta onde constavam as opções de suspeitos e não-suspeitos para cada exame apresentado e, sendo suspeitas, algumas opções diagnósticas para serem assinaladas (com os diagnósticos já referidos). As folhas de resposta foram computadas e analisadas estatisticamente sendo calculadas sensibilidade, especificidade, valor preditivo positivo, valor preditivo negativo, percentual global de concordância e índice Kappa conforme Landis e Koch (4) (Tabela 2). Também foi calculado o valor do chi-quadrado com nível de significância de 0,05 para a classificação global de suspeitos e não-suspeitos (Tabela 3).

Tabela 2. Classificação do índice Kappa conforme Landis e Koch (4)

\begin{tabular}{|c|c|}
\hline Indice de Карра & Classificação \\
\hline$<0,00$ & Quase inexistente \\
\hline $0,00-0,20$ & Pequena \\
\hline $0,21-0,40$ & Insatisfatória \\
\hline $0,41-0,60$ & Satisfatónia \\
\hline $0,61-0,80$ & Substancial \\
\hline $0,81-1,00$ & Quase perfeita \\
\hline
\end{tabular}

Nenhum observador referiu incapacidade em classificar o exame ou responder a folha de resposta. Também não houve qualquer queixa sobre a qualidade das imagens. Os resultados obtidos encontram-se distribuídos nas Tabelas 3 e 4 .

Tabela 3. Resultados de suspeitos e não-suspeitos (classificação global)

\begin{tabular}{lccc}
\hline \multicolumn{1}{c}{ Classificaçằo } & Suspeitos (padrão) & Nằ-suspeitos (padrăo) & Total \\
\hline Suspeitos (teste) & 145 & 61 & 206 \\
Não-suspeitos (teste) & 5 & 89 & 94 \\
Total & 150 & 150 & 300 \\
\hline
\end{tabular}

$\mathrm{P}<0,05$ e Kappa 0,56

Tabela 4. Sensibilidade, especificidade, concordância observada e valor Kappa segundo o diagnóstico nos casos considerados suspeitos

\begin{tabular}{lcccc}
\hline Diagnóstico & Sensibilidade & Especificidade & $\begin{array}{c}\text { Concordåncia } \\
\text { observada }\end{array}$ & Kappa \\
\hline Hérnia discal & 0,64 & 0,84 & 0,81 & 0,19 \\
Doença degenerativa discal & 0,58 & 0,84 & 0,79 & 0,15 \\
Discite infecciosa & 0,22 & 0,92 & 0,91 & 0,03 \\
Espondilartrose & 0,53 & 0,95 & 0,82 & 0,24 \\
\hline
\end{tabular}

A sensibilidade obtida da RM foi de 96,7 \% e sua especificidade foi 59,3\%. O valor preditivo positivo foi 70,4 \% e valor preditivo negativo foi 89,4\%. O percentual global de concordância (observada) foi 78 \%. O índice Kappa foi 0,56 que segundo Landis e Kock deve ser considerado satisfatório. 
Após análise de todas as respostas incluindo "suspeitos" e "não-suspeitos" e sendo suspeito, se houve acerto no diagnóstico etiológico, o índice de acerto global foi de $57 \%$, que equivale a 171 das 300 respostas. Sensibilidade, especificidade, percentual global de concordância (observada) e o índice kappa para todas as alterações encontradas (diagnósticos) são apresentados na Tabela 4.

\section{DISCUSSÃO}

A dor lombar afeta pelo menos dois terços da população adulta em algum período de suas vidas e está entre as dez principais causas de consulta médica (5). Além disso, é a causa mais comum de afastamento do trabalho nos Estados Unidos da América (5).

O diagnóstico etiológico das lombalgias ainda é tema de inúmeros estudos na literatura e se baseia no exame clínico ortopédico detalhado associado a exames de imagem como raios $\mathrm{x}$, discografia, mielografia, tomografia computadorizada e ressonância magnética (RM) (6).

A grande maioria dos pacientes com dor lombar melhora com tratamento sintomático, não sendo necessários diagnósticos precisos. Apenas $5 \%$ das lombalgias podem ser creditadas a causas neurológicas, sendo a hérnia discal responsável por $1 \%$ destes casos (6). O maior problema é distinguir pacientes com dor "benigna" daqueles que possuem doença de base ou alterações neurológicas, que conseqüentemente necessitarão de exames de imagem mais detalhados tais como ressonância magnética (RM) (3).

A RM não é necessária em todos pacientes com radiculopatia; de fato, ela está reservada àqueles casos em que a imagem irá guiar o tratamento, sendo útil em pacientes com sinais e sintomas neurológicos tais como claudicação e suspeita de estenose central ou foraminal (3). A despeito de sua indicação restrita, a solicitação deste exame chega a mais de $75 \%$ dos casos de lombalgia (2). Tendo em vista que o primeiro atendimento destes pacientes é dado por profissionais não-especializados, o aumento da prescrição de RM pode significar superestimação do seu verdadeiro valor. Isto ocasiona aumento demasiado e desnecessário do custo destes pacientes para o sistema de saúde e também induz erros diagnósticos e terapêuticos nos casos falso-positivos e/ou falsonegativos.

Nosso estudo evidenciou que quando avaliamos apenas a presença ou ausência de alterações, a RM apresenta sensibilidade de 96,7 \% e especificidade 
de 59,3 \% para doenças da coluna lombar. Entretanto, quando avaliamos a concordância de acertos nos diagnósticos, observamos baixos índices de Kappa. A sensibilidade observada para diagnóstico de hérnia discal foi 0,64, contudo sua concordância foi considerada "pequena" (Kappa de 0,19).

Estudo semelhante realizado por Thornbury et al (7) evidenciaram, após avaliação de exames de RM de 95 pacientes, sensibilidade para hérnias discais de 89 a $100 \%$, mas a especificidade foi de apenas 43 a $57 \%$. Janssen et al (8), num estudo de coorte com 180 pacientes, relataram sensibilidade de $96 \%$ e especificidade de $97 \%$, porém neste estudo o diagnóstico etiológico foi através da mielografia associada à RM.

Smith et al. (9) realizaram estudo comparativo entre imagens de RM da coluna lombar com doença degenerativa discal utilizando dois neuroradiologistas como observadores e obtiveram um índice de Kappa de 0,57. Em 1995, Raininko et al (10) realizaram estudo semelhante ao nosso e avaliaram variações estruturais nos discos intervertebrais de 122 imagens de RM da coluna lombar, torácica média e baixa utilizando como observadores um radiologista, um neuroradiologista e um cirurgião ortopédico. Obtiveram como resultado índice Kappa interobservador entre 0,47 a 0,61 na coluna lombar e torácica baixa, que foi classificado como satisfatório.

A grande maioria dos estudos semelhantes ao nosso revela índice Kappa que varia de 0,41 e 0,60 (classificado como satisfatório), entretanto estes estudos utilizaram observadores experientes e/ou métodos diagnósticos coadjuvantes em conjunto com a avaliação dos exames de RM. Nosso trabalho utilizou como observadores residentes e preceptores de ortopedia. Este fato revestese de grande importância posto que a maioria dos pacientes portadores de lombalgia é atendida por profissionais não-especialistas que aqui equivaler-seiam aos residentes. A despeito deste fato, obteve-se índice Kappa de 0,56 (considerado satisfatório) na diferenciação entre suspeito e não-suspeito, mas fica claro que a concordância no acerto diagnóstico foi muito baixa para todas as alterações apresentadas, incluindo-se hérnia discal. Isto pode indicar que não-especialistas são capazes de identificar alterações anatômicas na RM, entretanto não conseguem obter o correto diagnóstico.

O diagnóstico etiológico de lombalgia deve estar baseado em exame clínico bem conduzido que diferencie causas neurológicas e não-neurológicas ("benignas"). Os exames complementares devem ser solicitados com extrema 
cautela para evitar aumento desnecessário de custo e erros diagnósticos e terapêuticos. A RM tem alto valor diagnóstico quando há suspeita de lesão discal acompanhada ou não de lesão neural, contudo, deve ser solicitada e avaliada por profissionais comprovadamente experientes para evitar aumento de custos e conduta terapêutica inadequada. Posto que, a despeito de sua alta sensibilidade (96,7 \%), especialmente nos casos de doença discal, este exame apresenta baixa especificidade e mostra-se incapaz de fornecer diagnóstico etiológico correto nas mãos de observadores com experiência modesta.

Os autores concluem que a RM é exame de imagem com alta sensibilidade e moderada especificidade para as doenças da coluna lombar. Sua concordância interobservador é satisfatória para definir a presença de alteração anatômica, contudo, avaliadores com experiência modesta não conseguem identificar satisfatoriamente o correto diagnóstico etiológico. Por este motivo, acreditamos que a RM solicitada rotineiramente por não-especialistas ocasiona aumento desnecessário de custos e erros diagnósticos e terapêuticos freqüentes •

Conflito de interesse: Ninguno

\section{REFERENNCIAS}

1. Saal JS. General principles of diagnostic testing as related to painful lumbar spine disorders. Spine 2002;27:2538-45.

2. Stacey JA, Earl PS, Bryan RN, Bendebba M, Long DM. Trends in diagnostic for low back pain: has MR imaging been a substitute or add-on? Neuroradiology 1997;203:533-38.

3. Jarvik JG, Deyo RA. Diagnostic evaluation of low back pain with emphasis on imaging. Ann Intern Med 2002;137:586-97.

4. Landis JR, Kock GG. The measurement of observer agreement for categorical data. Biometrics 1997; 33:159-74.

5. Jarvik JG, Deyo RA. Imaging of lumbar intervertebral disk degeneration and aging, excluding disk herniations. Radiol Clin North Am 2000;38:1255-66.

6. Malanga GA, Nalder SF. Nonoperative treatment of low back pain. Mayo Clin Proc 1999;74:1135-48.

7. Thornbury JR, Fryback DG, Turski PA, Javid MJ, McDonald JV, Beinlich BR, et al. Disk-caused nerve compression in patients with acute low-back pain: diagnosis with MR, CT myelografy, and plain CT. Radiology 1993;186:731-8.

8. Janssen ME, Bertrand SL, Joe C, Levine MI. Lumbar herniated disk disease: comparison of MRI, myelografy, and post-myelografy CT scan with surgical finding. Orthopedics 1994;17:121-7. 
112 REVISTADE SALUD PÚBLICA · Volumen 10 (1), Febrero 2008

9. Smith BMT, Hurwitz, EL, Solsberg D, Rubinstein D, Corenman DS, Dwyer AP, et al. Interobserver reliability of detecting lumbar intervertebral disc high-intensity zone on magnetic resonance imaging and association of high-intensity zone with pain and anular disruption. Spine 1998;23:2074-80.

10. Raininko R, Manninen H, Battié MC, Gibbons LE, Gill K, Fisher LD. Observer variability in the assessment of disc degeneration on magnetic resonance images of lumbar and thoracic spine. Spine 1995;20:1029-35. 